\title{
Continuous Variable Entanglement of Orbital Angular Momentum States
}

\author{
Lassen, Mikael Østergaard; Leuchs, G.; Andersen, Ulrik Lund
}

Published in:

European Conference on Lasers and Electro-Optics 2009 and the European Quantum Electronics Conference. CLEO Europe - EQEC 2009.

Link to article, DOI:

10.1109/CLEOE-EQEC.2009.5191701

Publication date:

2009

Document Version

Publisher's PDF, also known as Version of record

Link back to DTU Orbit

Citation (APA):

Lassen, M. Ø., Leuchs, G., \& Andersen, U. L. (2009). Continuous Variable Entanglement of Orbital Angular Momentum States. In European Conference on Lasers and Electro-Optics 2009 and the European Quantum Electronics Conference. CLEO Europe - EQEC 2009. (pp. 1-1). IEEE. https://doi.org/10.1109/CLEOEEQEC.2009.5191701

\section{General rights}

Copyright and moral rights for the publications made accessible in the public portal are retained by the authors and/or other copyright owners and it is a condition of accessing publications that users recognise and abide by the legal requirements associated with these rights.

- Users may download and print one copy of any publication from the public portal for the purpose of private study or research.

- You may not further distribute the material or use it for any profit-making activity or commercial gain

- You may freely distribute the URL identifying the publication in the public portal 


\title{
Continuous Variable Entanglement of Orbital Angular Momentum States
}

\author{
M. Lassen ${ }^{1,2}$, G. Leuchs ${ }^{2,3}$, and U. L. Andersen ${ }^{1}$ \\ 1. Department of Physics, Technical University of Denmark, Fysikvej, 2800 Kgs. Lyngby, Denmark \\ 2. Max Planck Institute for the Science of Light, G"unther Scharowskystrasse 1, 91058 Erlangen, Germany \\ 3. University Erlangen-N"urnberg, Staudtstrasse 7/B2, 91058 Erlangen, Germany.
}

The simplest spatial mode that can carry orbital angular momentum (OAM) is the first order Laguerre-Gaussian (LG) mode which produce either a left-handed or right-handed corkscrew-like phase front and a ring-like intensity profile. In this contribution we present quadrature entanglement between the two first-order LG modes thereby demonstrating a new type of entanglement from non-degenerate OPOs. The entanglement is manifested in the squeezing of the rotated modes in the Hermite-Gauss (HG) basis, measured with a specially tailored local oscillator.

The most promising application of CV OAM states is their compatibility with atoms, thus allowing for storage of CV quantum information [1].
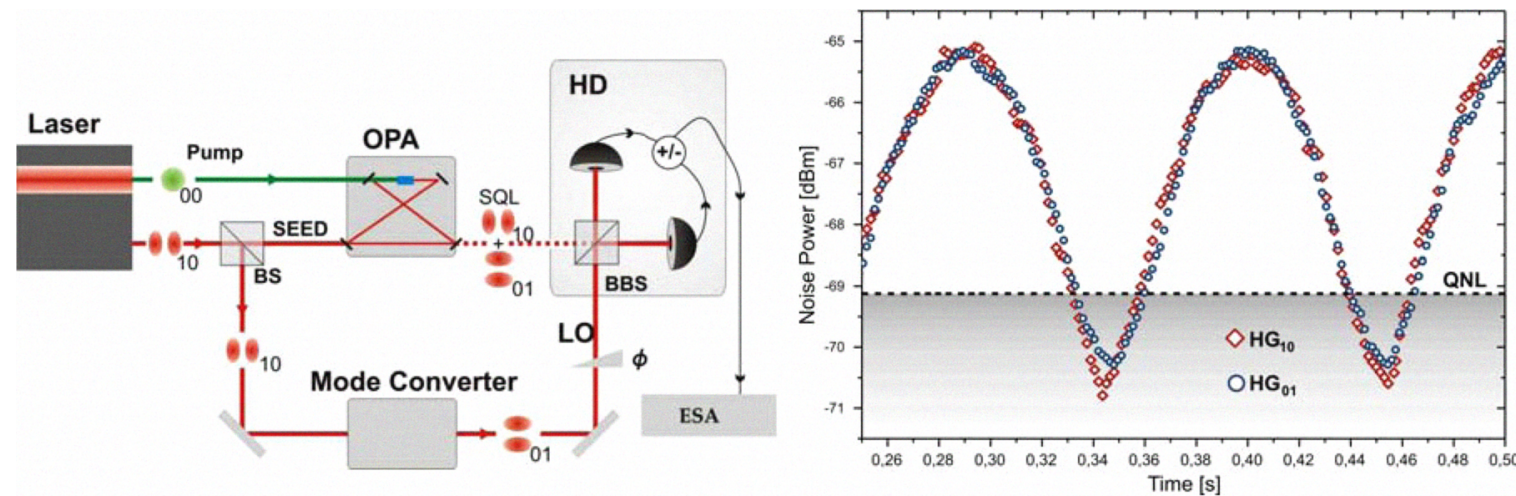

Fig. 1 Schematics of the experimental setup to generate amplitude squeezing. Experimental squeezing traces for the $\mathrm{HG}_{01}$ and $\mathrm{HG}_{10}$ modes, where the relative phase between the local oscillator and the squeezed beam is scanned. The traces are recorded with an ESA. Resolution bandwidth of $300 \mathrm{kHz}$ and a video bandwidth of $300 \mathrm{~Hz}$ at a detection frequency of $5.5 \mathrm{MHz}$. BBS: Balanced beam splitter (50/50 BS). PBS: Polarizing beam splitter. HWP: $(\lambda / 2)$ Half-wave plate. QWP: $(\lambda / 4)$ Quarter-wave plate.

Our OPO consist of a type I phase-matched nonlinear PPKTP crystal in a mode-stable optical cavity, where the polarization, the frequency and the spatial degree of freedom are usually degenerate, which lead to single mode quadrature squeezing as demonstrated by various groups. By optimizing the cavity resonance frequency it is possible to generate the two first order modes simultaneously. This is due to their frequency degeneracy (arising from their identical Gouy phase shifts). This has the consequence that the down-converted signal and idler photons are produced in two distinct orthogonal spatial modes (namely the OAM modes, $\mathrm{LG}^{+1}$ and $\mathrm{LG}^{-1}$ ), thus quadrature entanglement is generated between these two modes similarly to the production of entanglement between polarization modes.

Therefore to proof the existence of quadrature entanglement between the two LG modes, we measure the quadrature quantum noise of the spatial modes in a rotated basis composed of the first order $\mathrm{HG}$ modes, $\mathrm{HG}_{10}$ and $\mathrm{HG}_{01}$ [2]. The noise traces for the $\mathrm{HG}_{10}$ and $\mathrm{HG}_{01}$ modes, while the phase of the local oscillator is scanned, are depicted in Fig. 1. We measure amplitude quadrature squeezing of $-1.6 \mathrm{~dB}$ and $-1.4 \mathrm{~dB}$ for the $\mathrm{HG}_{10}$ and $\mathrm{HG}_{01}$ mode, respectively (corresponding to the minima of the squeezing traces). Inserting these values into the entanglement criterion we find [2]: $V\left(H G_{10}\right)+V\left(H G_{01}\right)=1.42<2$.

We thus have shown that the spatially non-degenerate OPO produces quadrature entanglement between the first order OAM modes.

In conclusion, we have generated a new quantum state of light composed of quadrature entangled LG modes. For the generation we used an OPO operating in a new regime where all field parameters are degenerate except for its spatial degree of freedom for which it is two-fold degenerate.

\section{References}

[1] R. Inoue, N. Kanai, T. Yonehara, Y. Miyamoto, M. Koashi and M. Kosuma, Phys. Rev. A 74, 053809 (2006).

[2] M. Lassen, G. Leuchs and U. L. Andersen, arXiv:0901.2783v1 [quant-ph] 19 Jan 2009 